\title{
Identification of Variants in the 4q35 Gene FAT1 in Patients with a Facioscapulohumeral Dystrophy-Like Phenotype
}

\author{
Francesca Puppo, ${ }^{1,2 \dagger}$ Eugenie Dionnet, ${ }^{1,2 \dagger}$ Marie-Cécile Gaillard, ${ }^{1,2}$ Pascaline Gaildrat, ${ }^{3}$ Christel Castro, ${ }^{1,2}$ Catherine Vovan, ${ }^{4}$ \\ Karine Bertaux ${ }^{4}$ Rafaelle Bernard, ${ }^{4}$ Shahram Attarian, ${ }^{1,2,5}$ Kanako Goto, ${ }^{6}$ Ichizo Nishino, ${ }^{6}$ Yukiko Hayashi, \\ Frédérique Magdinier, ${ }^{1,2}$ Martin Krahn, ${ }^{1,2,4}$ Françoise Helmbacher, ${ }^{8}$ Marc Bartoli, ${ }^{1,2,4 * \ddagger}$ and Nicolas Lévy ${ }^{1,2,4 \ddagger}$ \\ ${ }^{1}$ Aix Marseille Université, GMGF, Marseille, France; ${ }^{2}$ Inserm, UMR, S 910, Marseille, France; ${ }^{3}$ Institute for Research and Innovation in \\ Biomedecine (IRIB), Inserm, UMR 1079, University of Rouen, Rouen, France, ${ }^{4}$ Département de Génétique Médicale et de Biologie Cellulaire, \\ AP-HM, Hôpital d'Enfants de la Timone, Marseille, France; ${ }^{5}$ Department of Neurology and Neuromuscular Diseases, CHU La Timone, Marseille, \\ France, ${ }^{6}$ NCNP, National Institute of Neuroscience, Tokyo, Japan; ${ }^{7}$ Department of Neurophysiology, Tokyo Medical University, Tokyo, Japan; ${ }^{8}$ Aix \\ Marseille Université, CNRS, IBDM, UMR 7288, Marseille, France
}

Communicated by Mireille Claustres

Received 16 June 2014; accepted revised manuscript 11 January 2015.

Published online 23 January 2015 in Wiley Online Library (www.wiley.com/humanmutation). DOI: 10.1002/humu.22760

\begin{abstract}
Facioscapulohumeralmuscular dystrophy (FSHD) is linked to copy-number reduction $(N<10)$ of the $4 q$ D4Z4 subtelomeric array, in association with DUX4-permissive haplotypes. This main form is indicated as FSHD1. FSHD-like phenotypes may also appear in the absence of D4Z4 copy-number reduction. Variants of the SMCHD1 gene have been reported to associate with D4Z4 hypomethylation in DUX4-compatible haplotypes, thus defining FSHD2. Recently, mice carrying a musclespecific knock-out of the protocadherin gene Fat1 or its constitutive hypomorphic allele were shown to develop muscular and nonmuscular defects mimicking human FSHD. Here, we report FAT1 variants in a group of patients presenting with neuromuscular symptoms reminiscent of FSHD. The patients do not carry D4Z4 copy-number reduction, 4q hypomethylation, or SMCHD1 variants. However, abnormal splicing of the FAT1 transcript is predicted for all identified variants. To determine their pathogenicity, we elaborated a minigene approach coupled to an antisense oligonucleotide (AON) assay. In vitro, four out of five selected variants induced partial or complete alteration of splicing by creating new splice sites or modifying splicing regulators. AONs confirmed these effects. Altered transcripts may affect FAT1 protein interactions or stability. Altogether, our data suggest that defective FAT1 is associated with an FSHD-like phenotype.
\end{abstract}

Hum Mutat 36:443-453, 2015. (C) 2015 Wiley Periodicals, Inc.

KEY WORDS: neuromuscular pathology; facioscapulohumeral dystrophy; FAT1-protocadherin

\section{Introduction}

Facioscapulohumeralmuscular dystrophy (FSHD; MIM \#158900) [Justin Besancon et al., 1964; Balatsouras et al., 2007] is the third most common human neuromuscular disease. Symptoms mainly appear during the second decade of life and are characterized by progressive weakness of the facial, scapular, and humeral muscles, later spreading toward the lower limb muscles. Nonmuscular tissues are also frequently affected in FSHD; hearing loss manifests in $75 \%$ of patients, and retinal telangiectasia occurs in $60 \%$ of patients [Wohlgemuth et al., 2004]. Learning difficulties and epilepsy may also occur as atypical symptoms in subsets of severely affected children [Saito et al., 2007].

Genetically, FSHD is linked to the presence of a reduced copy number (1-10 copies) of a tandemly repeated 3.3-kb segment (D4Z4 macrosatellite) on chromosome 4q35, whereas in healthy individuals, this repeat varies from 11 to 100 copies [Wijmenga et al., 1990; van Deutekom et al., 1993]. This main form of FSHD, referred to as FSHD1, accounts for approximately $95 \%$ of cases [Richards et al., 2012]. Such a heterozygous D4Z4-array reduction is associated with chromatin relaxation on specific permissive haplotypes [Lemmers et al., 2002], which might result in stabilization of the DUX4 transcript, encoded by the retrogene located in D4Z4, and expression of this transcription factor [Gabriëls et al., 1999; Dixit et al., 2007; Lemmers et al., 2010; Spurlock et al., 2010; Vanderplanck et al., 2011]. A form of FSHD not linked to D4Z4 contraction accounts for 5\% of patients (contraction-independent FSHD) [van Overveld et al., 2003]. In a majority of these patients described at present, now referred to as FSHD2, variants of SMCHD1 (MIM \#614982; GenBank NM_015295.2) [Lemmers et al., 2012; Larsen et al., 2014; Lemmers et al., 2015], a gene known to play a role in chromatin relaxation and maintenance of $\mathrm{X}$ chromosome inactivation [Blewitt et al., 2008; Gendrel et al., 2012], have been reported to associate with D4Z4 hypomethylation in DUX4-compatible 4q haplotypes, thereby leading to DUX4 overexpression as well.

Nevertheless, large-scale studies performed on healthy individuals and FSHD patients have shown that the permissive haplotype (4qA161) on FSHD-sized repeats, although rare, is present in the healthy population, thus constituting a rare uncommon polymorphism [van Overveld et al., 2000; Lemmers et al., 2007; Scionti et al., $2012 a, 2012 b$ ] and indicating that DUX4 protein expression is not in itself sufficient to trigger FSHD symptoms. Furthermore, DUX4 RNA and protein have been detected, although at low levels, in muscle biopsies of healthy individuals carrying DUX4-compatible haplotypes in the absence of $4 \mathrm{q}$ contraction [Jones et al., 2012; Broucqsault et al., 2013]. This finding indicates that although necessary, DUX4 activation is not sufficient on its own to trigger the appearance 
of symptoms [Jones et al., 2012], implying the existence of modifier genes/mutations that contribute to this complex syndrome. As such, SMCHD1 variants were recently shown to constitute modifier alleles of FSHD1 [Sacconi et al., 2013; Larsen et al., 2014]. Therefore, evidence of cosegregation for multiple genetic factors began to accumulate, contributing to the genotype-phenotype correlation in a proportion of FSHD families. However, other cases of contractionindependent FSHD are not explained by SMCHD1/DUX4 synergy, leaving room for the identification of other genes involved in FSHD pathogenesis.

Recently, mouse models carrying a muscle-specific knock-out of the protocadherin gene Fat1 or its constitutive hypomorphic allele have been shown to develop defects in the shape and position of specific groups of muscles in the shoulder and face [Caruso et al., 2013]. The human FAT1 gene (MIM \#600976; GenBank NM_005245.3) is composed of 27 exons spanning a region of $139 \mathrm{~kb}$ and encodes a $506-\mathrm{kDa}$ transmembrane protein with 34 cadherin domains followed by a laminin and five EGF motifs in its extracellular part. Studies on the protocadherin Fatl demonstrated its influence on smooth muscle cell motility [Hou and Sibinga, 2009], actin accumulation at neuronal synapses [Moeller et al., 2004], the Hippo signaling pathway [Bennett and Harvey, 2006; Cho et al., 2006], and cell polarity [Skouloudaki et al., 2009]. While constitutive loss of FAT1 leads to a significant degree of perinatal lethality in mice [Ciani et al., 2003; Caruso et al., 2013], incomplete alterations of the FAT1 gene have been identified in rare developmental $4 \mathrm{q}$ syndromes characterized by mild facial dysmorphisms, abnormalities in skeletal and cardiac development, and growth and mental retardation [Ockey et al., 1967; Bendavid et al., 2007; Kitsiou-Tzeli et al., 2008]. Genetic links between FAT1 gene polymorphisms and susceptibility to bipolar disorder or schizophrenia have also been established [Blair et al., 2006; Abou Jamra et al., 2008; Jung and Jun 2013]. Finally, somatic mutations inducing high expression of FAT1 or its variant isoforms have been associated with both acute lymphoblastic leukemia [de Bock et al., 2012] and breast cancer progression [Lee et al., 2012], whereas other mutations (and CNV [copy number variation]) causing FAT1 protein loss of function are associated with glioblastoma, colorectal, or head and neck cancers [Morris et al., 2013a].

For the first time, in a study by Caruso et al. (2013), FAT1 was shown to control muscle patterning by modulating the polarity of myoblast migration during embryonic development, to be involved in regionalized muscle wasting, and to have a role in adult muscle fiber functions. Mice also exhibit extramuscular defects, such as retinal vasculopathy and abnormal inner ear patterning, which possibly represents a hearing impairment [Caruso et al., 2013]. Furthermore, misregulation of FAT1 expression was observed in fetal FSHD1 muscle tissue, and a CNV mapping in a putative regulatory enhancer of FAT1 preferentially segregated with FSHD-like patients who presented no D4Z4 contraction [Caruso et al., 2013]. Although FSHD-like symptoms only represent a subset of FAT lossof-function phenotypes, the map of muscles that show developmental impairment in FAT1 mutants strongly resembles one affected by human FSHD, raising the provocative hypothesis that FAT1 acts as a modifier gene to the disease.

Here, we sought to identify FAT1 variants in 49 Japanese cases affected by neuromuscular disease that closely resembles FSHD, according to the diagnostic criteria defined by the European Expert Group on FSHD [Padberg et al., 1991] and presenting no D4Z4 copy-number reduction in either $4 \mathrm{q} 35$ or 10q26, no reciprocal rearrangements [Yamanaka et al., 2004], no hypomethylation at D4Z4, and no SMCHD1 mutations. In 10 out of 49 cases analyzed, we identified 10 different variants of the FAT1 gene. To determine their pathogenic effect on FAT1 splicing, we elaborated a minigene approach, coupled to an antisense oligonucleotide (AON) assay. The in vitro results showed a partial or complete alteration of splicing either by creating a new acceptor splice site or by modifying splicing regulators in FAT1 variants. AONs confirmed the effect of these nucleotide substitutions. Altogether, our data suggest that a defective FAT1 protein may be associated with an FSHD-like phenotype.

\section{Methods}

\section{Study Subjects and Samples DNA Preparation; PCR and Sanger Sequencing Are Reported in the Supp. Methods}

\section{Bioinformatic predictions}

Amino acid substitutions were predicted for their impact on protein function by three online algorithms: polymorphism phenotyping v2 (Polyphen-2), SIFT, and prophyler (see Web links for references and Supp. Table S1 for results). PolyPhen-2 version 2.2.2 (http://genetics.bwh.harvard.edu/pph2) calculates the impact of amino acid substitutions using protein sequences from the UniProt database, structural data from PDB/DSSP, and comparative considerations from UCSC multiple alignments of 45 vertebrate genomes. For a mutation, PolyPhen-2 calculates the probability that this mutation is damaging and estimates a false-positive rate (the chance that the mutation is classified as damaging when it is in fact nondamaging). Based on the threshold of this rate, a mutation is qualitatively defined as benign, possibly damaging, or probably damaging. SIFT (v1.03, http://sift.jcvi.org) predictions are based on the degree of conservation of amino acid residues in sequence alignments derived from closely related sequences. The score ranges from 0 to 1 . The amino acid substitution is predicted to be damaging if the score is $\leq 0.05$. The impact of variants on FAT1 splicing was predicted by Human Splicing Finder (HSF; version 2.4.1) (cut-off for donor or acceptor sites is 55\%) [Desmet et al., 2009] and Alamut Visual 2.2, released in June 2012. This software integrates four different algorithms for splicing prediction: SpliceSiteFinder (http://www.umd.be/HSF/; cut-off for donor and acceptor sites 70\%), MaxEntScan (no cut-off provided), NN Splice (score for donor and acceptor ranges between 0 and 1, cut-off is 0.4 ), and GeneSplicer (no cut-off provided). For HSF, as well as for Alamut, the threshold for ESEFinder is the following: SF2/ASF, 1.956; SC35, 2.383; SRp40, 2.67; and SRp55, 2.676. Predictions were considered strong enough to be tested in a minigene reporter assay in the following cases: nucleotide substitutions that create or break regulatory ESE or SSE splicing sites or determine the creation of a new splicing site stronger than the natural one. The inclusion criteria for experimental analysis of variants also accounted for available data from dbSNP137 and included a minor allele frequency lower than 0.005 (Table 1; Supp. Table S1).

\section{Methylation analysis}

For sodium bisulfite sequencing, $2 \mu \mathrm{g}$ of genomic DNA was denatured for $30 \mathrm{~min}$ at $37^{\circ} \mathrm{C}$ in $0.4 \mathrm{M} \mathrm{NaOH}$ and incubated overnight in a solution of $3 \mathrm{M}$ sodium bisulfite, $\mathrm{pH}$, and $10 \mathrm{mM}$ hydroquinone using a previously described protocol [Ehrlich et al., 1982]. Converted DNA was then purified using the Wizard DNA CleanUp kit (Promega, Marseille, France) following manufacturer's recommendation and precipitated with ethanol. Modified DNA was amplified using the forward 5' -AAATATGTAGGGAAGGGTGTAAGTT- $3^{\prime}$ and 


\begin{tabular}{|c|c|c|c|c|c|c|c|c|c|c|c|c|c|}
\hline FAT1 CDS sequencing results & $\mathrm{J} 2$ & J6 & $\mathrm{J} 7$ & J15 & J16 & $\mathrm{J} 20$ & $\mathrm{~J} 21$ & $\mathrm{~J} 29$ & $\mathrm{~J} 41$ & J51 & Exon & $\begin{array}{c}\text { East Asian allele } \\
\text { count/allele } \\
\text { number }\end{array}$ & $\begin{array}{c}\text { General allele } \\
\text { count/allele } \\
\text { number }\end{array}$ \\
\hline c. $2215 \mathrm{~A}>$ G p.Met739Val & & & & & & & $\mathrm{X}$ & & & & 2 & $1 / 8892$ & $2 / 124670$ \\
\hline c. $3770 \mathrm{G}>$ A p.Arg1257Gln & & & & & $\mathrm{X}$ & & & & & & 5 & $12 / 8894$ & $22 / 124752$ \\
\hline c. $4358 \mathrm{G}>\mathrm{A}$ p.Arg $1453 \mathrm{His}$ & & $\mathrm{X}$ & & & & & & & & & 8 & $15 / 8724$ & $195 / 120308$ \\
\hline c. $4723 \mathrm{G}>$ A p.Ala1575Thr & & & $\mathrm{X}$ & $\mathrm{X}$ & & & & & & & 9 & 0 & 0 \\
\hline c.4959G>A p.Val1653Val & & & & & & $\mathrm{X}$ & & & & & 10 & $9 / 8878$ & $9 / 124606$ \\
\hline c.8963A>T p.Lys2988Ile & & & & & & & & $\mathrm{X}$ & & & 11 & $141 / 8888$ & $155 / 124660$ \\
\hline c. $8991 \mathrm{G}>$ A p.Thr2997Thr & $\mathrm{X}$ & & & & & & & & & & 11 & 0 & $20 / 124668$ \\
\hline c. $10331 \mathrm{~A}>\mathrm{G}$ p.Asn3444Ser & & & & & & & & & & $\mathrm{X}$ & 17 & $9 / 8890$ & $9 / 124578$ \\
\hline c.12051C>T p.Cys4017Cys & & & & & & & & & $\mathrm{X}$ & & 22 & 0 & $25 / 124654$ \\
\hline c. 13374 G>A p.Gln4458Gln & & & & & & & X & & & & 27 & $2 / 8894$ & $2 / 124730$ \\
\hline Age of onset & Birth & $\begin{array}{c}30- \\
50\end{array}$ & $>50$ & $<10$ & $\begin{array}{c}10- \\
>20\end{array}$ & & $20-30$ & $20>30$ & & $10>20$ & & & \\
\hline FSH & +++ & ++ & + & ++ & +++ & ++ & ++ & ++ & +++ & ++ & & & \\
\hline Lower limbs & ++ & & & + & & + & ++ & ++ & + & + & & & \\
\hline
\end{tabular}

$\mathrm{X}$ indicates the presence of mutation relative to cDNA sequence and relative to GenBank reference NM_005245.3. Nucleotide numbering uses +1 as the A of the ATG translation initiation codon in the reference sequence, with the initiation codon as codon 1 . Black shadow indicates validated effect on splicing, whereas gray one stresses potentially damaging amino acid changes. Last two columns represent minor allele count in East Asian and general healthy population, respectively, as reported by Version 0.2 ExAC (Exome Aggregation Consortium) database.

reverse 5'-GGAGAGAGGGTTTGGTATATTTAAG-3' primer set designed with the MethPrimer software [Bird 2002] to amplify 21 CpGs (275 bp) within the D4Z4 proximal region [Gaillard et al., 2014]. We avoided CpGs in the primer sequence in order to amplify methylated and unmethylated DNA with the same efficiency. Amplification was conducted using the High Fidelity Taq polymerase (Roche, Paris, France) according to manufacturer's instructions. After initial denaturation, the amplification conditions were the following: $94^{\circ} \mathrm{C}$ for $20 \mathrm{sec}, 54^{\circ} \mathrm{C}$ for $30 \mathrm{sec}, 72^{\circ} \mathrm{C}$ for $1 \mathrm{~min}$ for 10 cycles, and then the elongation was increased by $30 \mathrm{sec}$ at each subsequent cycle for 25 cycles. The PCR products were then purified using the Wizard SV gel and PCR Purification System (Promega) and cloned using the pGEM ${ }^{\circledR}$-T Easy Vector cloning kit (Promega). Randomly selected colonies were grown overnight at $37^{\circ} \mathrm{C}$ with ampicillin selection and PCR amplified directly using T7 and SP6 primers. At least 10 independent colonies were sequenced according to the Sanger method by Eurofins MWG Operon (Ebersberg, Germany) with either T7 or SP6 primers.

Sequences were analyzed using BiQ Analyzer software, and the average methylation score was calculated as the number of methylated CpGs for the total number of CpGs in the reference sequence (Table 2).

\section{Cell culture}

HEK293 cells (human embryonic kidney cell line) were grown in Dulbecco's modified Eagle medium (Gibco, Saint Aubin, France) supplemented with $15 \%$ fetal calf serum and $1 \%$ PSA (penicillin streptomycin actinomycin) in a $5 \% \mathrm{CO}_{2}$ atmosphere at $37^{\circ} \mathrm{C}$.

\section{Generation of constructs for splicing minigene reporter assay}

The splicing minigene assay has been previously described [Gaildrat et al., 2010]. DNA fragments corresponding to FAT1 wild-type and mutant exons surrounded by upstream and downstream intronic sequences were amplified from genomic DNA of patients heterozygous for the nucleotide substitution by using exon-specific forward and reverse primers (Supp. Table S2) carrying $5^{\prime}$ tails with BamHI (or BglII) and MluI restriction sites, respectively (underlined in Supp. Table S3). After digestion with BamHI (or BglII) and MluI, the PCR products were inserted into the BamHI and MluI cloning sites in the intron of the pCAS2 vector containing a two-exon splicing reporter minigene. The insert was then sequenced to identify the minigene constructs containing the FAT1 exon carrying the wild-type or substituted nucleotide and to ensure that no extra substitutions were added during amplification or cloning.

\section{Transfection and analysis of RT-PCR products}

The wild-type and mutant minigene constructs were transiently transfected into HEK293 cells using the promofectine transfection reagent, according to manufacturer's instructions (PromoKine, Heidelberg, Germany). Cells were collected $24 \mathrm{hr}$ post-transfection. Total RNA was extracted using the PureLink ${ }^{\circledR}$ RNA Mini Kit (Ambion; Life Technologies, Saint Aubin, France), according to the manufacturer's instructions, followed by a DNase treatment with the DNA-free Kit (Ambion; Life Technologies). The RT-PCR reactions were performed using the SuperScript OneStep RT-PCR with Platinium Taq kit (Life Technologies), according to the manufacturer's instructions, with $500 \mathrm{ng}$ RNA as a template in a $50-\mu 1$ reaction volume. Reactions were performed using $300 \mathrm{nM}$ of forward pCASKO1F (5'-TGACGTCGCCGCCCATCAC-3') and reverse pCAS2R primers ( $5^{\prime}$-ATTGGTTGTTGAGTTGGTTGTC-3') [Gaildrat et al., 2010]. The reverse transcription program had one cycle: $50^{\circ} \mathrm{C}$ for $30 \mathrm{~min}$. The PCR program had 35 cycles of amplification of $96^{\circ} \mathrm{C}$ for $45 \mathrm{sec}, 50^{\circ} \mathrm{C}$ for $45 \mathrm{sec}$, and $72^{\circ} \mathrm{C}$ for $1 \mathrm{~min}$. RT-PCR products were separated by electrophoresis on $2 \%$ agarose 1000 (Invitrogen, Saint Aubin, France) gels containing ethidium bromide and visualized by exposure to nonsaturating ultraviolet light.

\section{AON design and transfection}

AONs with full-length 2-O-methyl-substituted ribose moieties and phosphorothioate internucleotide linkages were purchased from Eurogentec (Angers, France). AONs were designed according to several criteria: sequences targeting exon 5 $\left(5^{\prime}-U^{*} U^{*} U^{*} C^{*} C^{*} C^{*} G^{*} C^{*} U^{*} C^{*} A^{*} G^{*} G^{*} G^{*} A^{*} G^{*} U^{*} C^{*} U^{*} G-3^{\prime}\right)$ and exon $17 \quad\left(5^{\prime}-G^{*} A^{*} C^{*} A^{*} C^{*} U^{*} G^{*} U^{*} A^{*} G^{*} U^{*} U^{*} U^{*} C^{*} C^{*} C^{*} C^{*} U^{*}\right.$ $\left.G^{*} G^{*} A-3^{\prime}\right)$ were designed complementarily to the wildtype sequence. In parallel, the AON sequence for exon 11 


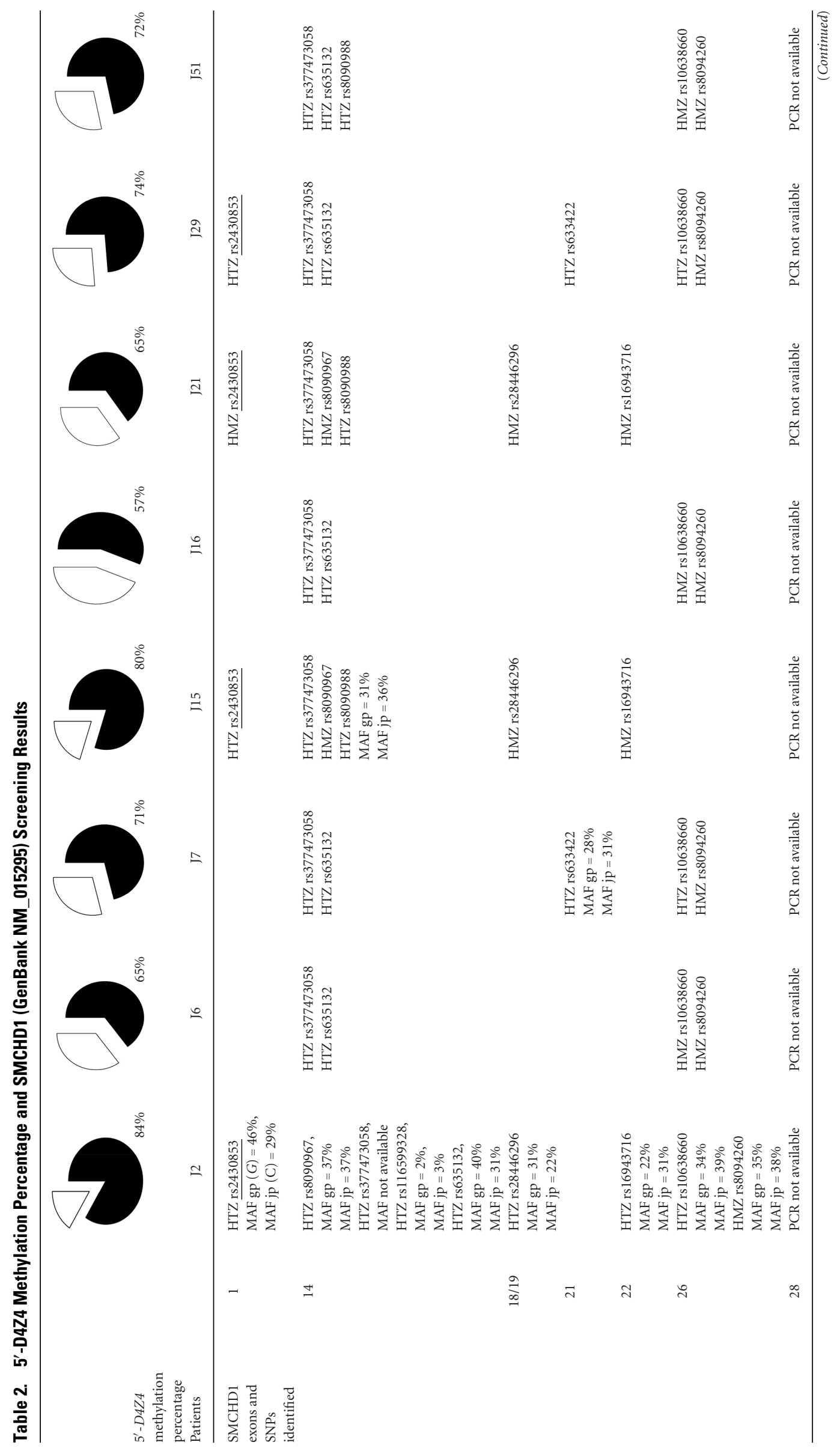




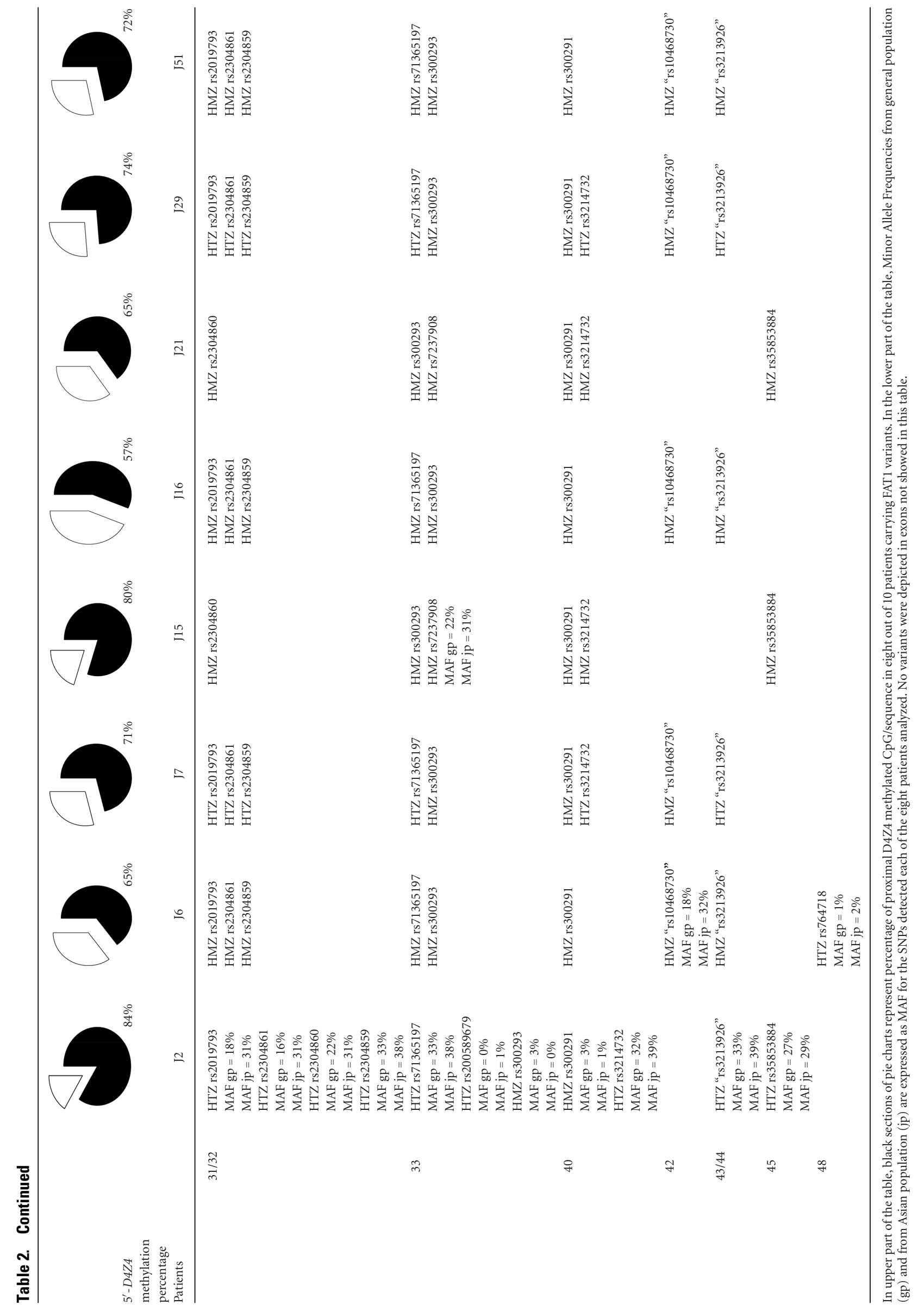


(5'-A* $\left.U^{*} C^{*} A^{*} G^{*} U^{*} U^{*} G^{*} C^{*} U^{*} G^{*} U^{*} G^{*} A^{*} U^{*} A^{*} G^{*} U^{*} A^{*} A-3^{\prime}\right)$ was designed complementarily to the variant sequence.

HEK293 cells $\left(4 \times 10^{5}\right.$ cells $)$ grown in 12-well plates to $80 \%$ confluence were transfected with $1.1 \mu \mathrm{g}$ of AON and $0.5 \mu \mathrm{g}$ of pCAS2 constructions using Lipofectamine 2000 reagent according to the manufacturer's instructions (Life Technologies). Total RNA was isolated from cultured cells $24 \mathrm{hr}$ after transfection, and RT-PCR analysis was performed as described above.

\section{Results}

In this work, 49 Japanese cases affected by neuromuscular disease were selected based on the homogeneity of muscles presenting clinical signs (Table 3 ) and closely resembling FSHD according to the diagnostic criteria defined by the European Expert Group on FSHD [Padberg et al., 1991]. No patients presented with D4Z4 copy-number reduction [Yamanaka et al., 2004]. The age distribution of patients with available clinical information was $60.8 \%$ of patients under the age of 30 at the time of disease presentation. The facial, scapular, and humeral muscles were simultaneously affected in $45.8 \%$ of patients, and $89.6 \%$ of them had at least two out of the three muscle groups affected. Progression of the disease toward the lower limbs was present in $72.9 \%$ of patients, with most affected at the proximal limb muscles. Other myopathies presenting with similar phenotypic appearances but specific histological defects were ruled out in 47 out of 49 individuals by muscular biopsy examination. Specifically, muscle from the J21 case showed scattered fibers with rimmed vacuoles. Protein analyses showed normal expression of dystrophin, sarcoglycans, dystroglycans, merosin, collagen 6, dysferlin, caveolin-3, calpain 3, and emerin. A sequence analysis of the GNE gene was normal (data not shown). Moreover, J51 was diagnosed with Nemaline myopathy based on the muscular biopsy (data not shown), whereas no variants in the ACTA1 gene were found [Wallefeld et al., 2006]. Finally, clinical data from other members of the patients' families were not available for any individual included in this study.

Among these patients, we found nucleotide substitutions in the FAT1 gene with a potentially damaging effect on transcript processing in 10 cases (Table 1; Supp. Tables S1 and S4). In particular, three of the substitutions have not been previously reported (c.4723G $>A$; c. $4959 \mathrm{G}>\mathrm{A}$ and c. $12051 \mathrm{C}>\mathrm{T}$ ). In all patients, the FAT1 variants were present on one allele and distributed in different exons without a specific mutation hotspot. The transition type of nucleotide substitution was overrepresented and corresponded to nine out of 10 nucleotide substitutions. The age of onset was under the age of 30 for six out of 10 patients carrying FAT1 nucleotide variants, and for two individuals, the age of symptom appearance was not available. The facial, scapular, and humeral muscles were simultaneously affected in three out of these 10 cases, and nine of them had at least two out of the three muscle groups affected. Progression of the disease toward the lower limbs was present in seven of the patients (details in Table 1).

Several publications have reported that D4Z4 hypomethylation, in the context of a DUX4-permissive haplotype associated with variants in the SMCHD1 gene, may cause FSHD2 and contribute to FSHD1 [Lemmers et al., 2012; Sacconi et al., 2013; Lemmers et al., 2015] with moderate but significant differences in the methylation level between asymptomatic carriers and individuals with clinical FSHD [Gaillard et al., 2014; Lemmers et al., 2015]. To determine whether variation in the FAT1 gene segregates with D4Z4 hypomethylation in our different cases, we used the sodium bisulfite sequencing method to measure the level of methylation of the 21
CpGs in the proximal D4Z4 region in eight out of the 10 patients carrying FAT1 variants. The results, reported in Table 3, show that less than $50 \%$ of CpGs displaying D4Z4 hypomethylation associated with the appearance of the muscular defects clinically similar to FSHD.

Furthermore, we screened and excluded SMCHD1 variants in all patients carrying nucleotide variants for FAT1 (Table 2; Supp. Table S5); thus, eight out of 10 FSHD-like patients do not carry any of the genetic features and epigenetic marks usually observed in FSHD1 and SMCHD1-linked FSHD2 patients.

Interestingly, for some of the FAT1 variants identified, the minor allele count in the East Asian and general population relative to the total allele number is available for 63,000 exomes by the Exome Aggregation Consortium (ExAC), Cambridge, MA (URL: http://exac.broadinstitute.org) [11/2014], as reported in Table 1. However, these variants are not reported as SNPs in any of the Japanese SNP databases that we could access (HAPMAP, KYUGEN JPK2 http://www.ncbi.nlm.nih.gov/projects/SNP/, and JSNP, http://snp.ims.u-tokyo.ac.jp/). The main criteria for inclusion in our functional investigations accounted for a frequency of the minor allele in the general population lower than 0.002 . The frequency of FAT1 SNPs that we found in our group of patients compared with the Japanese and general Caucasian population is indicated in Supp. Table S3.

Six out of 10 nucleotide substitutions that we observed lead to amino acid substitutions in different extracellular cadherin domains of FAT1. For all of the substitutions, anomalies in splicing of the FAT1 transcript have been predicted using Human Splicing Finder, HSF, and Alamut software (see methods for URLs), as detailed in Supp. Table S1 [Desmet et al., 2009]. The drastic loss of splicing regulator sites or the creation of new splicing sites stronger than the natural ones was predicted in seven cases. Five of the six amino acid substitutions were also predicted to have a deleterious impact on protein function by several algorithms (see Methods for URL references and Supp. Table S1 for detailed results).

To evaluate the splicing effect of selected FAT1 variants, we performed a functional assay based on a comparative analysis of the splicing pattern of wild-type and mutant sequences in the pCAS2 minigene-expressing vector [Gaildrat et al., 2010]. Five variants were selected based on their suitability for the experimental conditions of the test and the strength of in silico splicing predictions. Fragments corresponding to the FAT1 exons studied, surrounded by $150 \mathrm{bp}$ of upstream and downstream intronic sequences, were amplified from genomic DNA of patients and subcloned into the pCAS2 vector. After transient transfection in HEK293 cells, the splicing patterns and efficiency of wild-type and mutant FAT1 exon incorporation in the minigene were analyzed by RT-PCR and sequencing. As reported in Figure 1, we showed that four cases, c.3770G >A (pArg1257Gln), c.8963A>T (pLys2988Ile), c.8991G >A (pThr2997Thr), and c.10331A $>\mathrm{G}$ (pAsn3444Ser), result in partial or complete splicing defects. One defect may lead to nonsensemediated decay for c.8963A $>$ T, which implies a loss of frame of aberrant mRNA. In the other three cases, shorter half-lives of the aberrant mRNAs or deleterious forms of the translated FAT1 protein may be produced. In contrast to in silico predictions, no splicing effect in HEK293 cells was depicted for the c.4723G > A (p.Ala1575Thr) amino acid substitution, (Supp. Fig. S1) but in vivo tissue-specific splicing effects cannot be excluded. Among the nucleotide substitutions with a splicing effect in the functional minigene assay, the c.3770G $>$ A transition (identified in patient J16) induced complete skipping of exon 5 from the mutant transcript, as indicated in Figure 1a, suggesting an effect on splicing either by disrupting an exonic splicing enhancer (ESE) element and/or by creating an exonic 
(a)

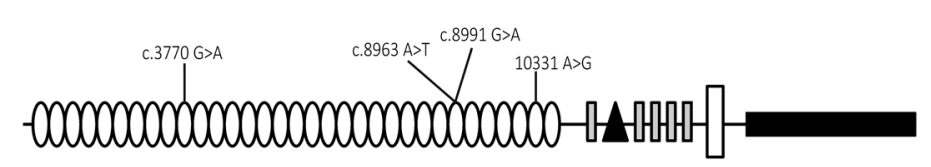

0 Cadherin domain; $\boldsymbol{\Delta}$ Laminin domain; [EGF motif; ]Transmembrane domain; $\boldsymbol{\square}$ Intracellular domain

(b)

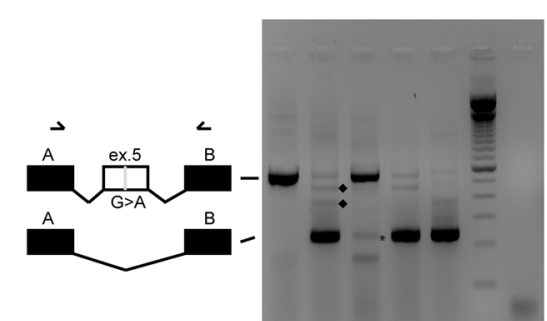

pFAT1 exon5 c.3770G (WT) + - + - - M BLNK

pFAT1 exon5 c.3770A (M) - + - + -

Empty pCAS minigene $\quad-\quad-\quad+$

AON C.3770G (WT) $\quad-\quad+++-$

Predictable FAT1

protein domains

affected by splicing (c)

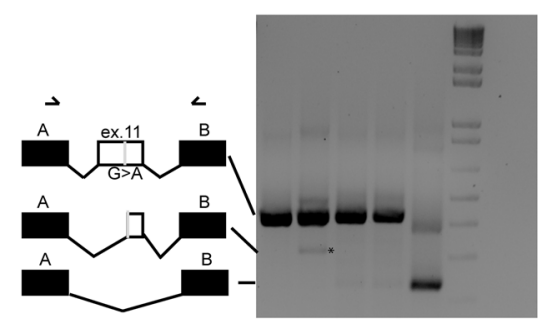

pFAT1 exon11 c.8991G (WT)+ - + - - M BLNK

pFAT1exon11 c.8991A (M) - + - + -

Empty pCAS minigene $\quad-\quad-\quad-+$

AON C.8991A (M) - $\quad-++$

Predictable FAT1

protein domains affected by splicing

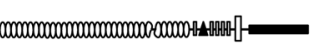

(d)

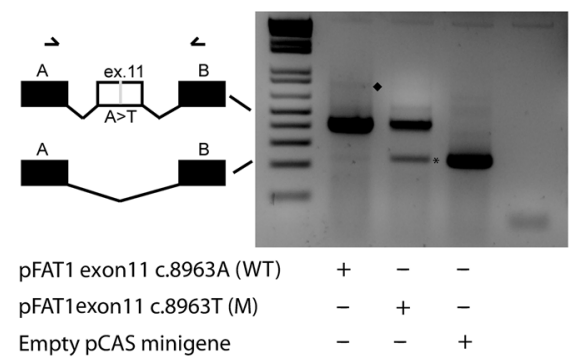

Predictable FAT1

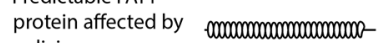
splicing
Position of the mutation (e)
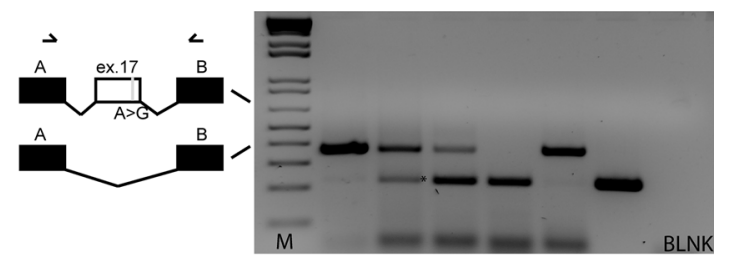

PFAT1 exon17 c.10331A (WT) + pFAT1 exon17 c.10331G (M)

Empty pCAS minigene AON c.10331A (WT)

AON c.3770G

Predictable FAT1

protein domains

affected by splicing

* Aberrant transcripts

- Aspecific PCR product

Figure 1. Abnormal splicing in FAT1-derived transcripts. A: Diagram representing FAT1 protein domains with the positions of the four variants tested by the minigene splicing assay coupled with the AON assay. Briefly, wild-type and mutant pCAS2 minigene constructs containing different FAT1 exons of interest were transfected into HEK293 cells alone or in combination with an AON specifically directed against the variant or wild-type allele. At $24 \mathrm{hr}$ after transfection, the splicing patterns of the minigenes were monitored by RT-PCR. All variants are expressed as the cDNA sequence and refer to GenBank NM_005245.3. B: Transcripts amplified from constructs containing wild-type (pFAT1 Exon5 c.3770G) and variant (pFAT1 Exon5 c.3770A) alleles of FAT1 exon 5 in the presence or absence of AON c.3770G and the empty pCAS2 minigene were separated on an agarose gel. The transcript amplified from the exon 5 variant construction containing exons from the pCAS2 minigene only is indicated by a star $(*)$. The effect of cotransfection of AON c.3770G and the wild-type exon 5 construct is presented together with a representation of the predicted effect on the protein. C: Agarose gel electrophoresis of transcripts produced from minigene constructs containing no insert (empty pCAS2 minigene), wild-type FAT1 exon 11 (pFAT1 Exon11 c.8991G), or mutant FAT1 exon 11 (pFAT1 Exon11 c.8991A) alone or cotransfected with AON c.8991A (mut). A star $\left({ }^{*}\right)$ indicates the transcript specifically produced from the variant exon 11 minigene and corresponding to 114 nucleotides deleted from exon 11. AON c.8991A rescued the exon 11 variant allele, allowing expression of the complete wild-type form of the exon. The potential effect on the FAT1 protein is also represented. D: Transcripts produced from constructs containing wild-type (pFAT1 Exon11 c.8963A) or variant (pFAT1 Exon11 c.8963T) FAT1 exon 11 and from the empty pCAS2 minigene were analyzed by agarose gel electrophoresis. Star $(*)$ indicates transcript amplified from exon 11 carrying nucleotide substitutions with complete skipping of it. Potential effect on the FAT1 truncated protein is reproduced. E: Agarose gel electrophoresis of transcripts produced from constructs containing wild-type (pFAT1 Exon17 c.10331A) or variant (pFAT1 Exon17 c.10331G) FAT1 exon 17 in the presence or absence of AONs (c.10331A, c.3770G) and the pCAS2 minigene. As indicated by a star (*), the transcript amplified from the exon 17 variant construct only contains exons from the pCAS2 minigene. The effect of cotransfection of AON c.10331A with the pFAT1 Exon17 c.10331G minigene is also presented together with the schema of the effect on the translated FAT1 protein. A0N c.3770G from exon 5 does not have a specific splicing effect on wild-type exon 17. 
Table 3. Summary of Neuromuscular Clinical Signs Found in Patients Presented in This Study

\begin{tabular}{|c|c|c|c|c|c|c|c|c|c|}
\hline \multirow[b]{2}{*}{ AGE at onset } & \multirow[b]{2}{*}{ Individuals } & \multirow[b]{2}{*}{$\%$} & \multicolumn{7}{|c|}{ Clinical details } \\
\hline & & & Facial & Scapular & Humeral & Abdominal & Proximal (lower limbs) & Distal (lower limbs) & Axial \\
\hline Birth/first decade & $11 / 46$ & $23.9 \%$ & $81.8 \%$ & $90.9 \%$ & $72.7 \%$ & $27.3 \%$ & $72.7 \%$ & $63.6 \%$ & $63.6 \%$ \\
\hline Second/third decade & $17 / 46$ & $36.9 \%$ & $58.8 \%$ & $94 \%$ & $88.2 \%$ & $23.5 \%$ & $76.5 \%$ & $64.7 \%$ & $47.1 \%$ \\
\hline Third/fifth decade & $10 / 46$ & $21.7 \%$ & $80 \%$ & $90 \%$ & $80 \%$ & $20 \%$ & $80 \%$ & $70 \%$ & $50 \%$ \\
\hline Older & $8 / 46$ & $17.4 \%$ & $50 \%$ & $75 \%$ & $75 \%$ & $25 \%$ & $50 \%$ & $25 \%$ & $37.5 \%$ \\
\hline Total with age and diagnosis available & 46 & & & & & & & & \\
\hline Total with diagnosis available & 48 & & $66.7 \%$ & $89.6 \%$ & $77.1 \%$ & $22.9 \%$ & $70.8 \%$ & $60.4 \%$ & $54.2 \%$ \\
\hline Facioscapulohumeral deficit & & & & $45.8 \%$ & & & & & \\
\hline Two off three muscles & & & & $89.6 \%$ & & & & & \\
\hline Progression to lower limbs & & & & & & & 72.9 & & \\
\hline
\end{tabular}

splicing silencer, as predicted by HSF and Alamut (Supp. Table S1). Interestingly, cotransfection with the AON carrying the wild-type sequence (c.3770G) induced a partial skipping of the exon, suggesting that this AON masks the predicted ESE element (Fig. 1a). The aberrant transcript missing exon 5 (RNA r.3643_3972del) produced by the variant allele would result, if translated, into an in-frame deletion of 110 amino acids at the protein level (p.Val1215_Ser1324del) with partial truncation of the cadherin 10 and 11 extracellular domains.

The second nucleotide substitution with an evident effect on splicing was the c.8963A $>\mathrm{T}$ transversion, located in exon 11 and identified in patient J29. The minigene assay showed the production of two transcripts: a primary one corresponding to normal exon inclusion and an aberrant minor transcript (5\% expression ratio versus the full-length transcript), which skips exon 11 (Fig. 1c). These results suggest that this variant alters splicing by disrupting ESE motifs, in agreement with the prediction (Supp. Table S1). If translated, the misspliced RNA (RNA r.8879_9075del) would result in a frameshift with the creation of a premature stop codon (p.Gly2960Asp*9) between exons 10 and 12 .

The third FAT1 variant, a c.8991G $>$ A transition, identified in patient $\mathrm{J} 2$, also induced an alteration in exon 11 splicing. Indeed, the results depicted in Figure $1 \mathrm{~b}$ show that the exon 11 minigene construct carrying the variant produces two transcripts, a primary one corresponding to the normal inclusion of the full-length exon and a second minor transcript (average expression ratio of $8 \%$ vs. the full-length transcript) in which the first 114 exonic nucleotides are deleted (RNA r.8879_8992del). This effect is in agreement with the Alamut and HSF predictions suggesting the creation of an acceptor splice site at position c.8992/c.8993 with a score slightly higher than the natural one (Supp. Table S1). We designed an AON (c.8991A) carrying the variant nucleotide to mask the created splicing acceptor site. Cotransfection of AON c.8991A and the variant minigene rescued normal splicing (Fig. 1b) and eliminated the aberrant transcript. This RNA would be translated in an in-frame deletion (p.Gly2960_Thr2997del) that corresponds to a truncation of cadherin domain 27 in the FAT1 protein.

Finally, the c.10331A $>\mathrm{G}$ transition, found in patient J51, induced a minor skipping of exon 17 (27\% average expression ratio vs. the full-length transcript) in the splicing minigene assay (Fig. 1d), and produced a transcript with 144 exonic nucleotides removed (RNA r.10207_10351del). This result could be the consequence of the predicted loss of an ESE element (Supp. Table S1) located in exon 17, thus interfering with the inclusion of exon 17 in the altered transcript. Transfection of AON c.10331A (wild type) with the wild-type exon 17 construct led to partial skipping, whereas in the presence of exon 17 carrying the nucleotide substitution, it leads to complete skipping of the exon (Fig. 1d). Finally, cotransfection of the AON specific for exon 5 (c.3770G) and a wildtype exon 17 construct did not interfere with exon 17 splicing. Thus, these results confirm that the wild-type AON specifically masks an element involved in exon inclusion. The main consequence of exon 17 skipping is likely the truncation of the cadherin 32 and 33 domains (p.Thr3403_Glu3451del) without the loss of the downstream reading frame.

Other nucleotide substitutions were also predicted to interfere with splicing, but they could not be easily tested, as they were not suitable for the conditions required for the minigene assay. In particular, patient J21 carries two different substitutions: c.2215A $>\mathrm{G}$ (p.Met739Val) and c.13374G $>$ A (p.Gln4458Gln). Testing these two variants using a minigene-based assay was not possible because of their location in FAT1 exons 2 and 27, respectively. As present, the parents' DNA is required to determine whether one of the two substitutions is a de novo substitution or if both substitutions are carried on the same allele. Both substitutions are predicted to affect splicing with equal strength, and an analysis of their respective impact on FAT1 transcription in the patient's biological samples, such as a muscle biopsy, would be interesting.

\section{Discussion}

In this study, we identified 10 different variants in the FAT1 gene in 10 out of 49 Japanese patients affected by an FSHD-like neuromuscular disease. The diagnosis was based on criteria defined by the European Expert Group on FSHD [Padberg et al., 1991]; the patients presented no D4Z4 copy-number reduction in either 4q35 or 10q26 and no reciprocal rearrangements [Yamanaka et al., 2004]. To rule out myopathies presenting similar phenotypic appearance but specific histological defects, a muscular biopsy examination was performed in 47 out of 49 individuals. For J21, the muscle biopsy showed nonspecific scattered fibers with rimmed vacuoles, and the J51 diagnosis of Nemaline myopathy was based on histological examination of his muscle biopsy (data not shown). In both cases, neither mutations in known genes nor expression defects in myopathyrelated proteins were observed. Thus, mutations in new genes responsible for the neuromuscular phenotype have not been ruled out. Interestingly, as the simultaneous presence of hypomethylation in D4Z4 regions in the context of a DUX4-permissive haplotype and variants in the SMCHD1 gene may cause FSHD2 and contribute to FSHD1 [Lemmers et al., 2012; Sacconi et al., 2013; Larsen et al., 2014; Lemmers et al., 2015], we measured the D4Z4 methylation level according to the methods and results recently published by our group [Gaillard et al., 2014]. In that publication, a significant reduction in the DNA methylation level at the D4Z4 proximal region was reported for individuals with clinical FSHD but not carrying a 
copy-number reduction (FSHD2) by sodium bisulfite sequencing, with a global level of methylated $\mathrm{CpG} /$ sequence below the threshold of 50\% [Gaillard et al., 2014]. In the group of patients investigated here, the average methylation level in the proximal D4Z4 region was above $50 \%$ (72\%), meaning that in these patients, FSHD symptoms are not associated with epigenetic changes at the $4 \mathrm{q} 35$ region (Table 2). Furthermore, no mutations in SMCHD1 were detected in any of the patients carrying nucleotide variants for FAT1 (Table 2). Even if the diagnosis of FSHD is based on the clinical evaluation of symptoms, genetic testing is required to confirm FSHD1 or FSHD2. However, a small proportion of patients with FSHD-like symptoms are not associated with D4Z4 copy-number reduction or with hypomethylated 4qA alleles and SMCHD1 variants, as reported here. In this situation, while the diagnosis may be questioned and reevaluated for some, it remains likely that the specific FSHD clinical signs might result from other genetic changes that affect processes also involved in FSHD. Identifying such genetic causes of FSHDlike cases would likely teach us about the biological mechanisms of this pathology. Thus, hypomorphic Fat1 mice presenting a FSHDlike phenotype [Caruso et al., 2013] and the identification of FAT1 variants in FSHD-like patients raise the challenging idea that FAT1 might be a disease gene associated with FSHD-like symptoms.

For some of the FAT1 variants depicted here, the minor allele count in the general and Japanese populations is reported, when available, in Table 1. Thus, we propose that these variants might represent very rare mutations that were possibly identified in presymptomatic individuals. Nonetheless, incomplete penetrance may not be excluded at this point. Consistently, a CNV located in a putative regulatory enhancer of FAT1 has previously been shown to preferentially segregate with FSHD in noncontracted FSHD-like patients, hence constituting the basis of tissue-specific alterations in FAT1 expression [Caruso et al., 2013].

The variants identified here fall into two categories that are not incompatible with each other. Six out of 10 variants led to amino acid substitutions localized in different extracellular cadherin domains of FAT1 and have been predicted to have a deleterious impact on protein function by several algorithms. Moreover, for all nucleotide substitutions, the drastic loss of splicing regulator sites or the creation of new splicing sites stronger than the natural ones have been suggested to give rise to partial or complete splicing defects. These defects would lead to shorter half-lives of aberrant mRNAs or the production of deleterious forms of the translated FAT1 protein. Thus, to demonstrate their pathogenic effect on FAT1 transcript splicing, we elaborated a minigene approach coupled to an $\mathrm{AON}$ assay. Some of these variants showed potential splicing alterations as well as deleterious amino acid changes, suggesting that both possibilities can occur; for example, any abnormally spliced mRNA, if translated, could result in a functionally aberrant protein. Therefore, only further experimental validation will determine the true functional relevance of each process. The FAT1 protein can be considered a "model protein," allowing us to correlate predictions to experimental findings. Moreover, the results shown here indicate that selected nucleotide substitutions have splicing effects in in vitro minigene assays. Interestingly, splicing defects might also depend on the chromosomal context or the presence of tissue-specific regulatory elements, which could only be analyzed in biological samples derived from patients. Nonetheless, our group recently showed that a minigene in vitro approach is reliable for confirming endogenous splicing defects (Kergourlay et al., 2014), supporting the idea that an actual damaging effect can be considered for variants showing an in vitro effect, even in the absence of in vivo confirmation.

In particular, the absence of an effect in c.4723G $>A$ (p.Ala1575Thr) (Supp. Fig. S1) does not exclude the impact of this variant on the endogenous transcript. This nucleotide substitution is strongly predicted to cause a deleterious amino acid substitution, as detailed in Supp. Table S1. To further validate the reliability of prediction algorithms at the experimental level, proteomic approaches as well as functional tests focusing on cadherin domain structure are in development. In the case of the c.3770G $>A$ (p.Arg1257Gln), c.8991G $>$ A (pThr2997Thr), and c.10331A >G (p.Asn3444Ser) transitions with a validated impact on splicing, aberrantly spliced mRNA isoforms may have more rapid turnover and shorter half-lives, thereby affecting intracellular FAT1 concentrations. The splicing effect is also compatible with an additional issue at the protein level because these aberrant transcripts, if translated, have been predicted to contribute to deleterious amino acid substitutions as well. For one of these variants (c.3770G>A), we showed that it completely prevents the in vitro inclusion of exon 5 in the transcript, thus producing an r.3643_3972del mRNA. If translated, the corresponding protein would lack the cadherin 10 and 11 extracellular domains without loss of the downstream reading frame. Nevertheless, the effect of this variant on splicing in vivo could be less drastic, allowing the partial integration of exon 5 carrying the variant nucleotide in the transcript. Thus, it would be interesting to investigate the importance of the arginine 1257 change to glutamine on FAT1 protein function as well as the potential dominant-negative effect due to the coexistence of multiple FAT1 protein-splicing isoforms in cells. Along the same line, c.10331A $>\mathrm{G}$ p.Asn3444Ser may contribute to two coexisting mRNA transcripts: the aberrant mRNA r.10207_10351del (p.Thr3403_Glu3451del) and the normal transcript, which may be translated into a FAT1 protein with a serine replacing the aspartic acid in position 3444. Even if these variants are compatible with FAT1 protein frame conservation, extracellular cadherin domains are expected to be lost and likely affect the stability and/or protein-protein interactions. Similar consequences may be predicted for c.8991G>A (pThr2997Thr) in which the aberrant mRNA r.8879_8992del, coexisting with the normal transcript, may be translated into a FAT1 protein truncated for cadherin domain 27. Interestingly, patient J29 carries a c.8963A $>\mathrm{T}$ transversion (p.Lys2988Ile) that is predicted to disrupt the ESE motifs Tra $2 \beta$ and 9G8 and to create a new hnRNP Al site, whereas amino acid substitution is not expected to have deleterious consequences on the protein. Here, we show the partial production of a variant transcript missing exon 11. The juxtaposition of exons 10 and 12 would create a frameshift in the FAT1 transcript and a premature stop codon 23 nucleotides after the beginning of exon 12 (p.Gly2960Asp*9). This incomplete FAT1 mRNA, if translated into a truncated FAT1 protein, may exert dominant-negative activity. Alternatively, the misspliced RNA (r.8879_9075del) may be eliminated by the nonsense-mediated mRNA decay pathway, causing haploinsufficiency reminiscent of the fat1 mouse model [Caruso et al., 2013].

AONs recognize and block special sequences in the neotranslated RNA that are otherwise bound by splicing protein complexes [Wein et al., 2010]. Based on the targeted splicing sequence, AONs may lead to the partial or total skipping of flanking exons. In our case, the AONs were designed to precisely recognize either the substituted or wild-type alleles, allowing us to demonstrate the specific effect of FAT1 alterations in vitro. Next, we will apply the AONs that mimic nucleotide substitutions to FAT1-expressing cells and animal models to characterize the functional consequences of these substitutions on the translated proteins. Moreover, for c.8991G $>$ A, the second minor transcript in which the first 114 exonic nucleotides were deleted, as observed using the minigene test, was rescued by cotransfection of AON c.8991A, which carries the variant nucleotide to mask the created splicing acceptor site. Animal models reproducing this variant are under development to evaluate the 
therapeutic consequences of $\mathrm{AON}$ injection and splicing rescue in vivo.

Some recent algorithms, which have been developed for functional annotation of genetic variants from high-throughput sequencing data, suggest that FAT1 is a dispensable human gene based on the number of stop codons identified in the study [Wang et al., 2010]. Interestingly, our study did not identify stop codons in any of the screened individuals or in healthy controls, whereas the constitutive loss of FAT1 function leads to perinatal lethality [Ciani et al., 2003; Caruso et al., 2013]. Thus, in independent functional and genetic studies, the partial preservation of FAT1 function would still be compatible with life. Accordingly, defective forms of the protein would have tissue-specific impacts and would be exerted at low doses by a dominant-negative effect. Hence, altered FAT1 would play a pathogenic role by affecting only specific interactions with protein partners and only during specific stages of development. Nevertheless, consistent with mice carrying the tissue-specific knock-out of FAT1 or its constitutive hypomorphic allele [Caruso et al., 2013], we cannot rule out haploinsufficiency as the pathogenic mechanism, at least for some of the variants reported here.

In addition to our present results, other germline mutations in the FAT1 gene may lead to developmental defects in subtle pathologies, such as schizophrenia and bipolar disorder susceptibility [Ockey et al., 1967; Blair et al., 2006; Bendavid et al., 2007; Abou Jamra et al., 2008; Kitsiou-Tzeli et al., 2008; Jung and Jun 2013]. Similarly, somatic mutations contribute to $\mathrm{Wnt} / \beta$-catenin pathway misregulation and cancer progression in specific tissues by both overexpression and inactivation of FAT1 protein function [de Bock et al., 2012; Lee et al., 2012; Morris et al., 2013a, 2013b]. Thus, aberrant FAT1 expression or incomplete or complete loss of function cause defects in tissues unrelated to FSHD, indicating that FAT1 has pleiotropic implications that may lead to a range of clinical consequences, with only a subset of those sharing similarities with FSHD. Thus, we propose the existence of a heterogeneous pathological entity, named FATopathy.

In perspective, adding FAT1 to the panel of neuromuscular disease-causing genes routinely tested for molecular diagnosis will be needed both to investigate the broader significance of FAT1 in disease pathogenesis and to better define the implication of multiple genetic interactions in neuromuscular disease appearance.

\section{Conclusion}

According to the evidence from the mouse model recently published and the identification of the CNV located in a transcriptional enhancer of the human FAT1 gene, which segregates in noncontracted FSHD-like patients, our genetic data further strengthen the link between the FAT1 gene and FSHD-like neuromuscular diseases.

\section{Acknowledgments}

The authors would like to thank all patients for their participation. We thank Christophe Pécheux, Mohamed Mesrati, and Cécile Mouradian for technical assistance. All the samples explored in this study were prepared by the Center of Biological Resources, Department of Medical Genetics, la Timone, France. We thank Vincent Meyer, Emmanuelle Salort-Campana, and Pr Jean Pouget for medical and scientific discussion. Jacques Beckmann and Isabella Ceccherini are warmly acknowledged for their assistance in reading the manuscript and their critical comments and suggestions. The funders had no role in the study design, data collection and analysis, decision to publish, or preparation of the manuscript.

\section{References}

Abou Jamra R, Becker T, Georgi A, Feulner T, Schumacher J, Stromaier J, Schirmbeck F, Schulze TG, Propping P, Rietschel M, Nöthen MM, Cichon S. 2008. Genetic variation of the FAT gene at $4 \mathrm{q} 35$ is associated with bipolar affective disorder. Mol Psychiatry 13:277-284.

Balatsouras DG, Korres S, Manta P, Panousopoulou A, Vassilopoulos D. 2007. Cochlear function in facioscapulohumeral muscular dystrophy. Otol Neurotol 28:7-10.

Bendavid C, Pasquier L, Watrin T, Morcel K, Lucas J, Gicquel I, Dubourg C, Henry C, David V, Odent S, Levêque J, Pellerin I, et al. 2007. Phenotypic variability of a 4q34->qter inherited deletion: MRKH syndrome in the daughter, cardiac defect and Fallopian tube cancer in the mother. Eur J Med Genet 50:66-72.

Bennett FC, Harvey KF. 2006. Fat cadherin modulates organ size in Drosophila via the Salvador/Warts/Hippo signaling pathway. Curr Biol CB 16:2101-2110.

Bird A. 2002. DNA methylation patterns and epigenetic memory. Genes Dev 16: 6-21.

Blair IP, Chetcuti AF, Badenhop RF, Scimone A, Moses MJ, Adams LJ, Craddock N, Green E, Kirov G, Owen MJ, Kwok JBJ, Donald JA, et al. 2006. Positional cloning, association analysis and expression studies provide convergent evidence that the cadherin gene FAT contains a bipolar disorder susceptibility allele. Mol Psychiatry 11:372-383.

Blewitt ME, Gendrel A-V, Pang Z, Sparrow DB, Whitelaw N, Craig JM, Apedaile A, Hilton DJ, Dunwoodie SL, Brockdorff N, Kay GF, Whitelaw E. 2008. SmcHD1, containing a structural-maintenance-of-chromosomes hinge domain, has a critical role in X inactivation. Nat Genet 40:663-669.

de Bock CE, Ardjmand A, Molloy TJ, Bone SM, Johnstone D, Campbell DM, Shipman KL, Yeadon TM, Holst J, Spanevello MD, Nelmes G, Catchpoole DR, et al. 2012. The Fat 1 cadherin is overexpressed and an independent prognostic factor for survival in paired diagnosis-relapse samples of precursor B-cell acute lymphoblastic leukemia. Leukemia 26:918-926.

Broucqsault N, Morere J, Gaillard M-C, Dumonceaux J, Torrents J, Salort-Campana E, Maues De Paula A, Bartoli M, Fernandez C, Chesnais AL, Ferreboeuf M, Sarda L, et al. 2013. Dysregulation of 4q35- and muscle-specific genes in fetuses with a short D4Z4 array linked to facio-scapulo-humeral dystrophy. Hum Mol Genet 22:4206-4214.

Caruso N, Herberth B, Bartoli M, Puppo F, Dumonceaux J, Zimmermann A, Denadai S, Lebossé M, Roche S, Geng L, Magdinier F, Attarian S, et al. 2013. Deregulation of the protocadherin gene FAT1 alters muscle shapes: implications for the pathogenesis of facioscapulohumeral dystrophy. PLoS Genet 9:e1003550.

Cho E, Feng Y, Rauskolb C, Maitra S, Fehon R, Irvine KD. 2006. Delineation of a Fat tumor suppressor pathway. Nat Genet 38:1142-1150.

Ciani L, Patel A, Allen ND, ffrench-Constant C. 2003. Mice lacking the giant protocadherin mFAT1 exhibit renal slit junction abnormalities and a partially penetrant cyclopia and anophthalmia phenotype. Mol Cell Biol 23:3575-3582.

Desmet F-O, Hamroun D, Lalande M, Collod-Béroud G, Claustres M, Béroud C. 2009. Human Splicing Finder: an online bioinformatics tool to predict splicing signals. Nucleic Acids Res 37:e67.

van Deutekom JC, Wijmenga C, van Tienhoven EA, Gruter AM, Hewitt JE, Padberg GW, van Ommen GJ, Hofker MH, Frants RR. 1993. FSHD associated DNA rearrangements are due to deletions of integral copies of a $3.2 \mathrm{~kb}$ tandemly repeated unit. Hum Mol Genet 2:2037-2042.

Dixit M, Ansseau E, Tassin A, Winokur S, Shi R, Qian H, Sauvage S, Mattéotti C, van Acker AM, Leo O, Figlewicz D, Barro M, et al. 2007. DUX4, a candidate gene of facioscapulohumeral muscular dystrophy, encodes a transcriptional activator of PITX1. Proc Natl Acad Sci USA 104:18157-18162.

Ehrlich M, Gama-Sosa MA, Huang LH, Midgett RM, Kuo KC, McCune RA, Gehrke C. 1982. Amount and distribution of 5-methylcytosine in human DNA from different types of tissues of cells. Nucleic Acids Res 10:2709-2721.

Gabriëls J, Beckers MC, Ding H, De Vriese A, Plaisance S, van der Maarel SM, Padberg GW, Frants RR, Hewitt JE, Collen D, Belayew A. 1999. Nucleotide sequence of the partially deleted D4Z4 locus in a patient with FSHD identifies a putative gene within each $3.3 \mathrm{~kb}$ element. Gene 236:25-32.

Gaildrat P, Killian A, Martins A, Tournier I, Frébourg T, Tosi M. 2010. Use of splicing reporter minigene assay to evaluate the effect on splicing of unclassified genetic variants. Methods Mol Biol Clifton NJ 653:249-257.

Gaillard M-C, Roche S, Dion C, Tasmadjian A, Bouget G, Salort-Campana E, Vovan C, Chaix C, Broucqsault N, Morere J, Puppo F, Bartoli M, et al. 2014. Differential DNA methylation of the D4Z4 repeat in patients with FSHD and asymptomatic carriers. Neurology 83:733-742.

Gendrel A-V, Apedaile A, Coker H, Termanis A, Zvetkova I, Godwin J, Tang YA, Huntley D, Montana G, Taylor S, Giannoulatou E, Heard E, et al. 2012. Smchd1-dependent and -independent pathways determine developmental dynamics of CpG island methylation on the inactive X chromosome. Dev Cell 23:265-279.

Hou R, Sibinga NES. 2009. Atrophin proteins interact with the Fatl cadherin and regulate migration and orientation in vascular smooth muscle cells. J Biol Chem 284:6955-6965. 
Jones TI, Chen JCJ, Rahimov F, Homma S, Arashiro P, Beermann ML, King OD, Miller JB, Kunkel LM, Emerson CP, Wagner KR, Jones PL. 2012. Facioscapulohumeral muscular dystrophy family studies of DUX4 expression: evidence for disease modifiers and a quantitative model of pathogenesis. Hum Mol Genet 21:4419-4430.

Jung Y-E, Jun T-Y. 2013. Association between FAT gene and schizophrenia in the Korean population. Clin Psychopharmacol Neurosci 11:67-71.

Justin Besancon L, Pequignot H, Contamin F, Delavierre P, Rolland P. 1964. [Myopathy of the landouzy-d'ejerine type. Report of a historical case]. Sem Hôp 40:29902999.

Kergourlay V, Raï G, Blandin G, Salgado D, Béroud C, Lévy N, Krahn M, Bartoli M. 2014. Identification of splicing defects caused by mutations in the dysferlin gene. Hum Mutat 35:1532-41.

Kitsiou-Tzeli S, Sismani C, Koumbaris G, Ioannides M, Kanavakis E, Kolialexi A, Mavrou A, Touliatou V, Patsalis PC. 2008. Distal del(4) (q33) syndrome: detailed clinical presentation and molecular description with array-CGH. Eur J Med Genet 51:61-67.

Larsen M, Rost S, El Hajj N, Ferbert A, Deschauer M, Walter MC, Schoser B, Tacik P, Kress W, Müller CR. 2014. Diagnostic approach for FSHD revisited: SMCHD1 mutations cause FSHD2 and act as modifiers of disease severity in FSHD1. Eur J Hum Genet. doi:10.1038/ejhg.2014.191 [Epub ahead of print]

Lee S, Stewart S, Nagtegaal I, Luo J, Wu Y, Colditz G, Medina D, Allred DC. 2012. Differentially expressed genes regulating the progression of ductal carcinoma in situ to invasive breast cancer. Cancer Res 72:4574-4586.

Lemmers RJLF, Goeman JJ, van der Vliet PJ, van Nieuwenhuizen MP, Balog J, VosVersteeg M, Camano P, Ramos Arroyo MA, Jerico I, Rogers MT, Miller DG, Upadhyaya M, et al. 2015. Inter-individual differences in CpG methylation at D4Z4 correlate with clinical variability in FSHD1 and FSHD2. Hum Mol Genet 24:659-669.

Lemmers RJLF, de Kievit P, Sandkuijl L, Padberg GW, van Ommen G-JB, Frants RR, van der Maarel SM. 2002. Facioscapulohumeral muscular dystrophy is uniquely associated with one of the two variants of the 4q subtelomere. Nat Genet 32:235236.

Lemmers RJLF, Tawil R, Petek LM, Balog J, Block GJ, Santen GWE, Amell AM, van der Vliet PJ, Almomani R, Straasheijm KR, Krom YD, Klooster R, et al. 2012. Digenic inheritance of an SMCHD1 mutation and an FSHD-permissive D4Z4 allele causes facioscapulohumeral muscular dystrophy type 2. Nat Genet 44:1370-1374.

Lemmers RJLF, van der Vliet PJ, Klooster R, Sacconi S, Camaño P, Dauwerse JG, Snider L, Straasheijm KR, van Ommen GJ, Padberg GW, Miller DG, Tapscott SJ, et al. 2010. A unifying genetic model for facioscapulohumeral muscular dystrophy. Science 329:1650-1653.

Lemmers RJLF, Wohlgemuth M, van der Gaag KJ, van der Vliet PJ, van Teijlingen CMM, de Knijff P, Padberg GW, Frants RR, van der Maarel SM. 2007. Specific sequence variations within the $4 \mathrm{q} 35$ region are associated with facioscapulohumeral muscular dystrophy. Am J Hum Genet 81:884-894.

Moeller MJ, Soofi A, Braun GS, Li X, Watzl C, Kriz W, Holzman LB. 2004. Protocadherin FAT1 binds Ena/VASP proteins and is necessary for actin dynamics and cell polarization. EMBO J 23:3769-3779.

Morris LGT, Kaufman AM, Gong Y, Ramaswami D, Walsh LA, Turcan Ş, Eng S, Kannan K, Zou Y, Peng L, Banuchi VE, Paty P, et al. 2013a. Recurrent somatic mutation of FAT1 in multiple human cancers leads to aberrant Wnt activation. Nat Genet 45:253-261.

Morris LGT, Ramaswami D, Chan TA. 2013b. The FAT epidemic: a gene family frequently mutated across multiple human cancer types. Cell Cycle 12:1011-1012.

Ockey CH, Feldman GV, Macaulay ME, Delaney MJ. 1967. A large deletion of the long arm of chromosome no. 4 in a child with limb abnormalities. Arch Dis Child 42:428-434. van Overveld PGM, Lemmers RJFL, Sandkuijl LA, Enthoven L, Winokur ST, Bakels F, Padberg GW, van Ommen G-JB, Frants RR, van der Maarel SM. 2003. Hypomethylation of D4Z4 in 4q-linked and non-4q-linked facioscapulohumeral muscular dystrophy. Nat Genet 35:315-317.

van Overveld PG, Lemmers RJ, Deidda G, Sandkuijl L, Padberg GW, Frants RR, van der Maarel SM. 2000. Interchromosomal repeat array interactions between chromosomes 4 and 10: a model for subtelomeric plasticity. Hum Mol Genet 9:2879-2884.

Padberg GW, Lunt PW, Koch M, Fardeau M. 1991. Diagnostic criteria for facioscapulohumeral muscular dystrophy. Neuromuscul Disord 1:231-234.

Richards M, Coppée F, Thomas N, Belayew A, Upadhyaya M. 2012. Facioscapulohumeral muscular dystrophy (FSHD): an enigma unravelled? Hum Genet 131:325-340.

Sacconi S, Lemmers RJLF, Balog J, van der Vliet PJ, Lahaut P, van Nieuwenhuizen MP, Straasheijm KR, Debipersad RD, Vos-Versteeg M, Salviati L, Casarin A, Pegoraro E, et al. 2013. The FSHD2 gene SMCHD1 is a modifier of disease severity in families affected by FSHD1. Am J Hum Genet 93:744-751.

Saito Y, Miyashita S, Yokoyama A, Komaki H, Seki A, Maegaki Y, Ohno K. 2007. Facioscapulohumeral muscular dystrophy with severe mental retardation and epilepsy. Brain Dev 29:231-233.

Scionti I, Fabbri G, Fiorillo C, Ricci G, Greco F, D’Amico R, Termanini A, Vercelli L, Tomelleri G, Cao M, Santoro L, Percesepe A, et al. 2012a. Facioscapulohumeral muscular dystrophy: new insights from compound heterozygotes and implication for prenatal genetic counselling. J Med Genet 49:171-178.

Scionti I, Greco F, Ricci G, Govi M, Arashiro P, Vercelli L, Berardinelli A, Angelini C, Antonini G, Cao M, Di Muzio A, Moggio M, et al. 2012b. Large-scale population analysis challenges the current criteria for the molecular diagnosis of fascioscapulohumeral muscular dystrophy. Am J Hum Genet 90:628-635.

Skouloudaki K, Puetz M, Simons M, Courbard J-R, Boehlke C, Hartleben B, Engel C, Moeller MJ, Englert C, Bollig F, Schäfer T, Ramachandran H, et al. 2009. Scribble participates in Hippo signaling and is required for normal zebrafish pronephros development. Proc Natl Acad Sci USA 106:8579-8584.

Spurlock G, Jim H-P, Upadhyaya M. 2010. Confirmation that the specific SSLP microsatellite allele 4qA161 segregates with fascioscapulohumeral muscular dystrophy (FSHD) in a cohort of multiplex and simplex FSHD families. Muscle Nerve 42:820-821.

Vanderplanck C, Ansseau E, Charron S, Stricwant N, Tassin A, Laoudj-Chenivesse D, Wilton SD, Coppée F, Belayew A. 2011. The FSHD atrophic myotube phenotype is caused by DUX4 expression. PloS One 6:e26820.

Wallefeld W, Krause S, Nowak KJ, Dye D, Horváth R, Molnár Z, Szabó M, Hashimoto K, Reina C, De Carlos J, Rosell J, Cabello A, et al. 2006. Severe nemaline myopathy caused by mutations of the stop codon of the skeletal muscle alpha actin gene (ACTA1). Neuromuscul Disord 16:541-547.

Wang K, Li M, Hakonarson H. 2010. ANNOVAR: functional annotation of genetic variants from high-throughput sequencing data. Nucleic Acids Res 38:e164.

Wein N, Avril A, Bartoli M, Beley C, Chaouch S, Laforêt P, Behin A, Butler-Browne G, Mouly V, Krahn M, Garcia L, Lévy N. 2010. Efficient bypass of mutations in dysferlin deficient patient cells by antisense-induced exon skipping. Hum Mutat 31:136-142.

Wijmenga C, Frants RR, Brouwer OF, Moerer P, Weber JL, Padberg GW. 1990. Location of facioscapulohumeral muscular dystrophy gene on chromosome 4. Lancet 336:651-653.

Wohlgemuth M, van der Kooi EL, van Kesteren RG, van der Maarel SM, Padberg GW. 2004. Ventilatory support in facioscapulohumeral muscular dystrophy. Neurology 63:176-178.

Yamanaka G, Goto K, Ishihara T, Oya Y, Miyajima T, Hoshika A, Nishino I, Hayashi YK. 2004. FSHD-like patients without 4q35 deletion. J Neurol Sci 219:89-93. 